\title{
Supervised/ Unsupervised Classification of LULC using remotely Sensed Data for Coimbatore city, India
}

\author{
Y.Babykalpana \\ Research Scholar, \\ Anna University, \\ Coimbatore,India.
}

\author{
Dr. K.ThanushKodi \\ Director, Akshya College of Engineering \\ \& Technology, \\ Coimbatore,India
}

\begin{abstract}
The Landuse / Landcover system espoused by almost all Organisations and scientists, engineers and remote sensing community who are involved in mapping of earth surface features, is a system which is derived from the united States Geological Survey (USGS) LULC classification system. The application of RS and GIS involves determining of homogeneous zones, trend analysis of land use integration of new area changes or change detection etc.,
\end{abstract}

National Remote Sensing Agency(NRSA) Govt. of India has devised a generalized LULC classification system respect to the Indian conditions based on the various categories of Earth surface features, resolution of available satellite data, capabilities of sensors and present and future applications.

The profusion information of the earth surface offered by the high resolution satellite images for remote sensing applications. Using change detection methodologies to extract the target changes in the areas from high resolution images and rapidly update geodatabase information processing.

Traditionally, classification approaches have focused on per-pixel technologies. Pixels within areas assumed to be automatically homogeneous are analyzed independently. These new sources of high spatial resolution image will increase the amount of information attainable on land cover. Significance is that the data can be acquired by our eyes and the energy can be analyzed. But satellites are capable of collecting data beyond the visible band also
However, the traditional method of change detection are not suitable for high resolution remote sensing images. To overcome the limitations of traditional pixel-level change detection of high resolution remote sensing images, based on georeferencing and analysis method, this paper presents a unsullied way of multi-scale amalgamation for the high resolution remote sensing images change detection. Experiment shows that this method has a stronger advantage than the traditional pixel-level method of high resolution remote sensing image change detection.

General terms Landcover, Landuse, Analysis, Indian conditions

Keywords: Espouse, LULC classification, profusion information, multi-scale amalgamation, change detection, Remote sensing images. 


\section{INTRODUCTION}

A variety of change detection methods have beendeveloped now days. Some of the most common methods are (i). Image deferencing (2). Principal component analysis, (3). Post-classification comparison, (4). Change vector analysis (5). Thematic change analysis.

Traditionally, classification approaches have focused on per-pixel technologies. [1]Pixels within areas assumed to be automatically homogeneous are analyzed independently. These new sources of high spatial resolution image will increase the amount of information attainable on land cover[2].

The LULC classification system is designed according to the following criteria:-

1. The minimum level of interpretation accuracy using remotely sensed data should be at least $85 \%$.

2. The accuracy of interpretation for the several categories should be about equal. For this paper 2003 and 2008 data have been taken for classifying.

3. Repeatable results should be obtainable from one interpreter to another and from one time of sensing to another

4. The classification system should be applicable over extensive areas.

5. The categorization should permit land use to be inferred form the land cover types.

6. The classification system should be suitable for use with remote sensor data obtained at different times of the year.

7. Categories should be divisible into more detailed sub categories that can be obtained from largescale imagery or ground surveys.

8. Aggregation of categories must be possible

9. Compression with future land use land over data should be possible.

10. Multiple uses of land should be remitted when possible.

For efficient planning and management, the classified data in a timely manner, in order to get the classified data of the ground; satellites are the best resources to provide the data in a timely manner.
Fig 1.1. Overview - Process for Landuse and Landcover

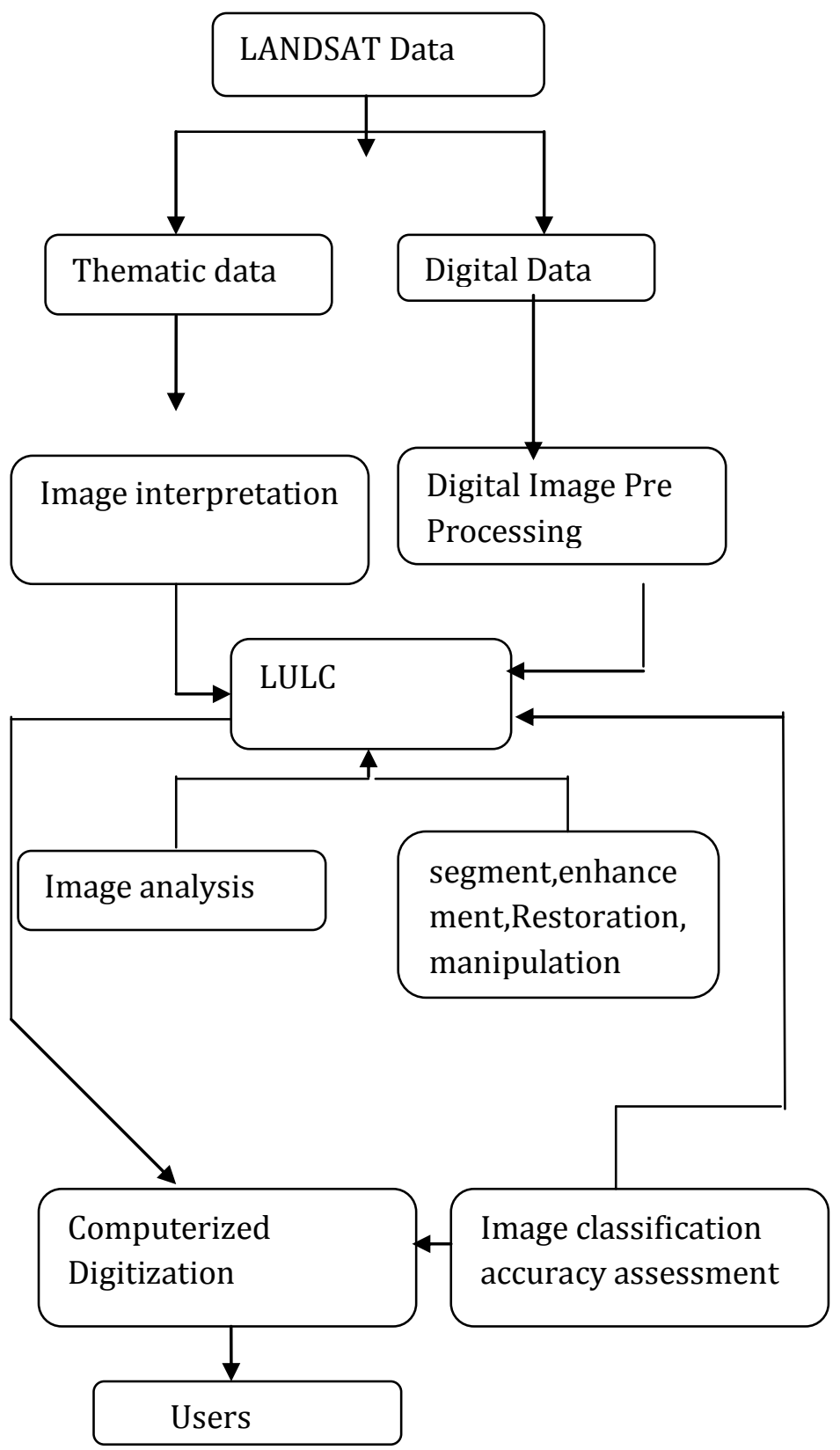

Significance is that the data can be acquired by our eyes (see'fig 1.1)and the energy can be analyzed. But satellites are capable of collecting data beyond the visible band also. This will help us to analyze the new things which are not possible in visible band[3].

\section{STUDY AREA GENERAL INFORMATION}

AREA: 105.5 KM2 (41 SQ MI)

Population: 1.5Million (2009 Census)

LATITUDE N: $10^{\circ} 10^{\prime}$ AND $11^{\circ} 30^{\prime}$ 
LONGITUDE: E $76^{\circ} 40^{\prime}$ AND $77^{\circ} 30^{\prime}$

Altitude: $43.2 \mathrm{M}$

CLOTHING: LIGHT CoTtons

Language Spoken: Tamil, English, Telugu and

Malayalam

Climate: Tropical

Temperature Range (DEg C):

Summer: MaX 41ㄷ, Min 38C

WINTER: MAX 32.8ㄷ, MIN 20.7으

RAINFALL: 92.2 MM

Fig 1.2 Remotely sensed image - TN

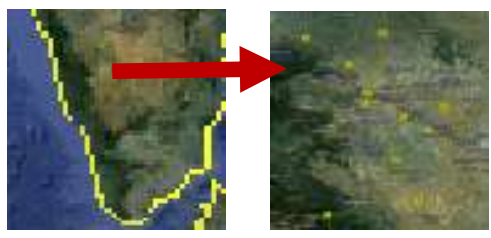

\section{METHODOLOGY}

Normally, the process includes edge detection techniques, image acquisition, image enhancement, segmentation classification, data modeling etc.

\section{Image acquisition}

Numerous electromagnetic and some ultra sonic sensing devices frequently are arranged in an array format.(See fig.1.2) CCD sensors are used in digital cameras and other light sensing instruments [8].

\subsection{Preprocessing}

To correct distorted or degraded image data to create a more faithful representation of the original scene, image rectification and restoration process is necked which is always termed as preprocessing.

\subsubsection{Edge detection}

An edge is a set of connected pixels that his on the boundary between two regions, Edge detection is performed on the image by the construction of edge detection operators like sobel edge detection, laplacian edge operator etc. For a continuous image $f(x, y)$, where $x$ and $\mathrm{y}$ are the row and column coordinates respectively, consider $2 \mathrm{D}$ derivatives $\delta \mathrm{yf}(\mathrm{x}, \mathrm{y})$ and $\delta \mathrm{xf}(\mathrm{x}, \mathrm{y})$. Two functions can be expressed :

1. Gradient magnitude

$|\Delta \mathrm{f}(\mathrm{x}, \mathrm{y})|=\sqrt{ }\left(\partial_{\mathrm{x}} \mathrm{f}(\mathrm{x}, \mathrm{y})\right)^{2}+\left(\partial_{\mathrm{y}} \mathrm{f}(\mathrm{x}, \mathrm{y})\right)^{2}$

2. Gradient orientation

$\left\llcorner\Delta \mathrm{f}(\mathrm{x}, \mathrm{y})=\operatorname{ArcTan}\left(\partial_{\mathrm{y}} \mathrm{f}(\mathrm{x}, \mathrm{y}) / \partial_{\mathrm{x}} \mathrm{f}(\mathrm{x}, \mathrm{y})\right)\right.$
Local maxima of the gradient magnitude identify edges in $f(x, y)$. The first derivative achieves a maximum and the second derivative is zero. For this reason, an alternative edge detection strategy is to locate zeros of the second derivatives of $\mathrm{f}(\mathrm{x}, \mathrm{y})[2]$.

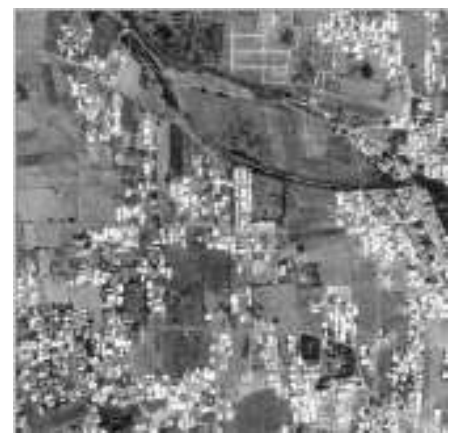

\section{Fig 1.3. edge detection sample $\mathbf{o} / \mathbf{p}$}

\section{Image segmentation technique}

Image segmentation is the partition and pick-up of the homogeneous regions of image. In the results of segmentation, the consistancy of gray the smoothing of boundary and the connectivity are fulfilled. The classical method of segmentation is the spatial cleaning based on the measurement space[1].

Image segmentation is crucial processing procedure for the classifications and feature extraction of high resolution remote sensing image[5]. The segmentation result is able to sway the effect of subsequent processing. At present the main image segmentation methods are

1. Thresh-hole based

2. Edge-band

3. Region-based

The edge-based segmentation is taken into account which is namely grounded on discontinuity of gray-level in imagery. The image is segmented by the edge of the different homogenous areas[4].

Adopting this method, the accuracy of edge positioning is high whereas the consecutive edge composed of a serial of unique pixels cannot produced, so a sequent process including bulky the detected edge points should he requisite.

\section{Clustering technique}

There are numerous clustering algorithms that can be used to determine the natural spectral groupings present in a data set. One common form of clustering, called the "k-means" approach accepts from the analyst the no. of clusters to be located in the data[6]. 
The algorithms then arbitrarily "seeds" or locates that number of clusters centers in the multidimensional measurement space[8]. Each pixel in the image is then assigned to the cluster whose arbitrary mean vector is closet.

\section{UNSUPERVISED CLASSIFICATION}

The family of classifiers involves algorithms that examine etc unknown pixels in an image and aggregate them into a number of classes based on the natural groupings or clusters present in the image values.

The basic premise is that values with in a given cover type should be close together in the measurement space; where as data in different classes should be comparatively well separated.

The classification algorithm is designed to automatically said dense regions within the n-dimensional hyper spectral data cloud[7]. The algorithm is based on the well-known observation that spectra of large, distinct land covers tend to cluster around a mean spectrum.

This is the basis for unsupervised classification parcel on cluster analysis[2]. The pixel density around the mean spectrum depends on the spectral variability of the land cover and the areas extent of the land cover.
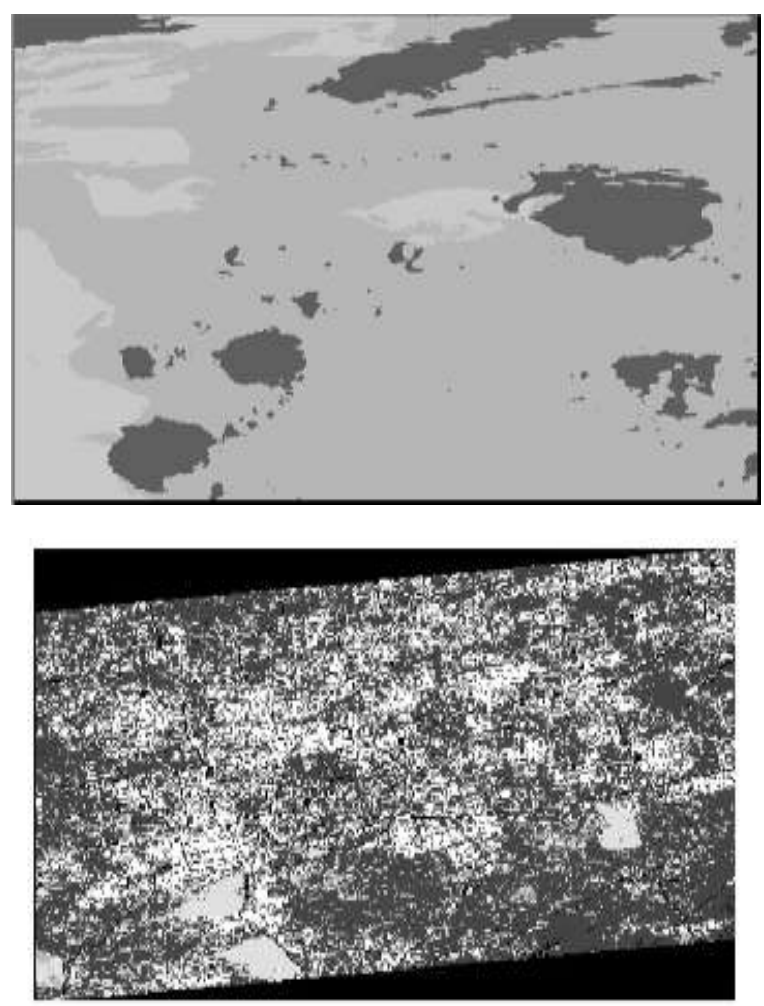

Fig. 5.1. Geo Referencing
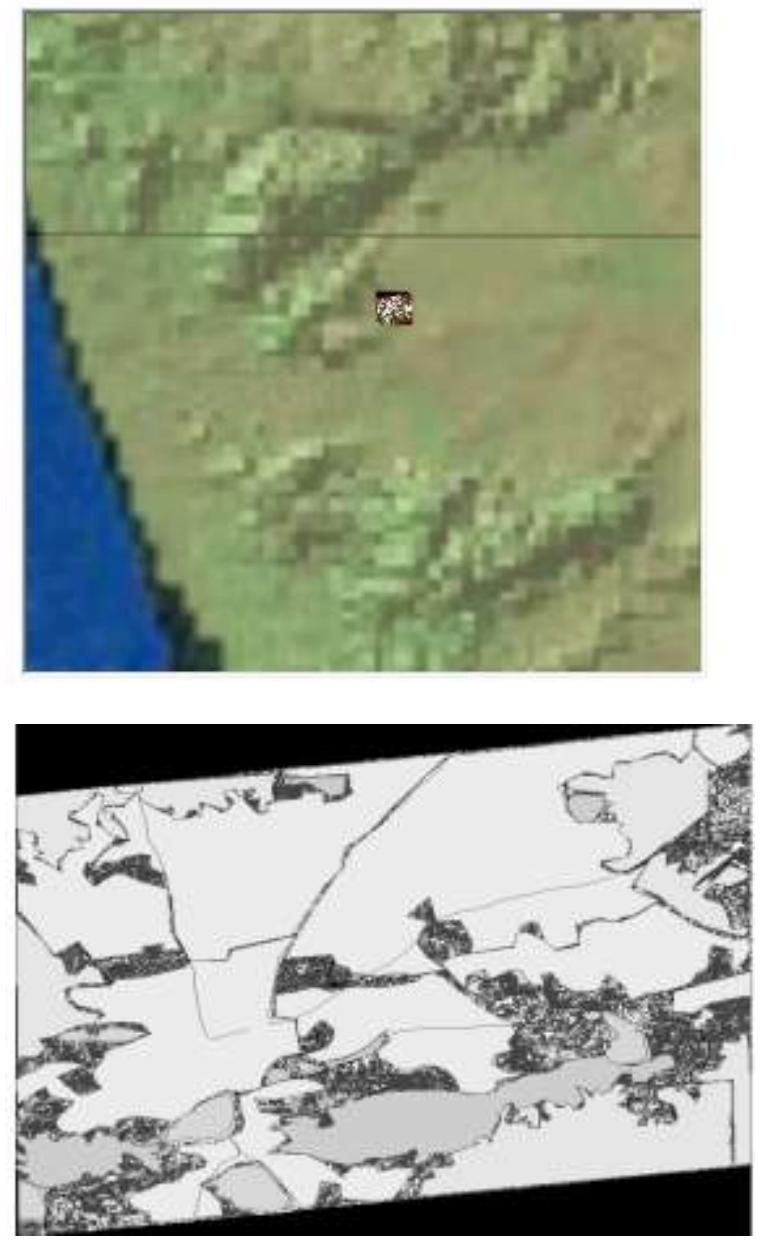

Fig. 5.2. Classification of Coimbatore city

\section{CONClusion}

Sample data

\begin{tabular}{||l|l|l||}
\hline Category & Hectares & Land Use \\
\hline \hline 1 & 177 & Water \\
\hline 2 & 210 & Vegetation \\
\hline \hline 3 & 90 & Urban/Edge \\
\hline
\end{tabular}




\begin{tabular}{||l||l||l|}
\hline 4 & 210 & Grassland \\
\hline \hline 5 & 120 & Bare Soil \\
\hline \hline 6 & 170 & Forest \\
\hline \hline- & 977 & Total \\
\hline
\end{tabular}

Fig 6.1. Report generated on Land Use

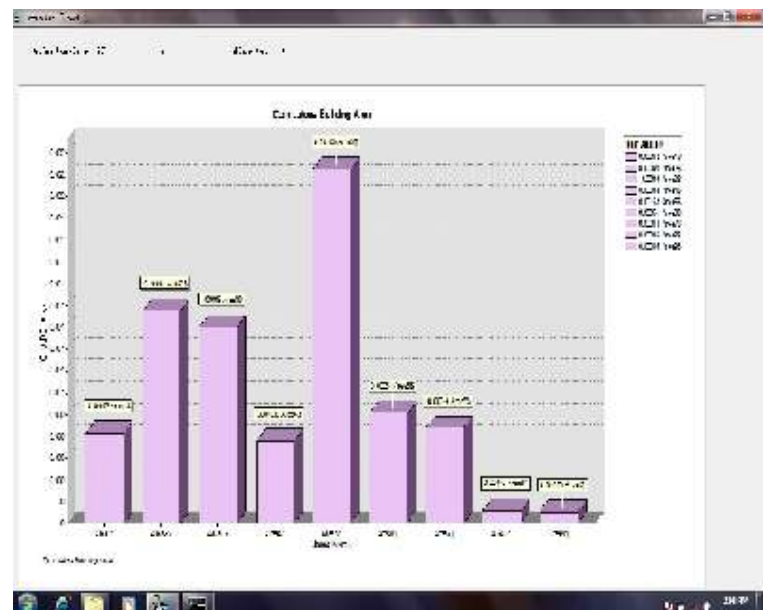

\section{Fig. 6.2 Urban Area Report}

The remote sensing data have been analyzed to fixed the land cover classification of our city, and to know how the use of land changes according to time and also performed the temporal analysis to analyze[3] all these things, the unsupervised classification method is used.This is very fast and useful analysis method. It is widely used for the crops[10] classification in the world and this classification method is used for land cover and land use because vegetation components are important in the images[9].The basic axis is also to preserve the greenery of the city for the healthy environment.

\section{References}

[1]. Jifen Liu, Maoting Gao, “ An Unsupervised Classification Scheme Using PDDP method for network Intrusion Detection", 978-0-7695-3497-8/08, IEEE transactions 2008,vol 8, pp. 658-662.

[2]. John Cipar,R.L.Wood, T.Cooley," Testing an Automated Unsupervised Classification Algorithm with Diverse Land covers", 1-4244-1212-9/07,IEEE Transactions 2007, vol.1, pp. 2589-2592.

[3]. LI.Jia-cun, QIAN Shao-meng, CHEN Xue," Object Oriented Method of Land Cover Change Detection Approach Using High Spatial Resolution Remote Sensing Data", vol 3, IEEE transactions 2003, pp. 3005-3007.

[4]. S.K.Srivastava, H.K.Singh, A.K.Sinha , " Agriculture land estimation from Satellite Images Using Hybrid Intelligence: A case study on Meerut City", IJCSKE, Vol 3, no1, Serials publications, Jan-June'09.

[5]. Thomas M.Liiesand, R.W.Kiefer, " Remote sensing and Image Interpretation", $4^{\text {th }}$ edition, John Wiley \& sons , Inc., pp. 473-488, 532-558.

[6]. Yokoyama, Kurosu T., SHI., and Fujita, M., 2001: Land use classification with textural analysis and the aggregation technique using multi-temporal JERS-1 L band SAR images. International Journal of Remote Sensing, 22, No. 4, pp. 595 -613.

[7].http://www.csc.noaa.gov/crs/lca/faq_gen.html\#WIRS

[8].http://www.eicinformation.org/data/requestform

[9].http://www.gisdevelopment.net/tutorials/software/index .htm

[10]. Institute Of remote sensing , Anna university, Chennai, India.

\section{Acknowledgement}

The authors are grateful to the Director, IRS, Anna university, Chennai who helped in this excellent work. The authors are thankful to Anna university Research centre delegates who helped them in al this work since 2007. 